\title{
Study on Composite Modified Ceramic Shell of Mineralizer and Short-Cut Carbon Fiber
}

\author{
Xiang-Feng MENG ${ }^{1, a}$, Yu-Tao ZHAO ${ }^{1, b^{*}}$, Zhi-Hong JIA ${ }^{1, c}$, Xiang-Feng LIANG ${ }^{1, d}$ \\ ${ }^{1}$ School of Materials Science and Engineering, Jiangsu University, Zhenjiang 212013, China \\ amengxiangfeng1693@163.com, b'zhaoyt@ujs.edu.cn, jjia_zh@126.com, d848824666@qq.com \\ ${ }^{*}$ Corresponding author
}

Keywords: Investment Casting, Mineralizer, Short-Cut Carbon Fiber, High-Temperature Strength, Gravitational Distortion.

\begin{abstract}
This experiment by using the composite method of the Mineralizer and short-cut carbon fiber to improve the performance of the shell, The influence of addition of mineralizer and the addition and length of short-cut carbon fiber on the high-temperature strength and high-temperature gravitational distortion of ceramic shell was investigated by orthogonal experiment.The results showed that the composite modified fused corundum shell is $11.33 \mathrm{MPa}$ higher than the normal fused corundum shell of $4.43 \mathrm{MPa}$, and the high temperature gravitational distortion is reduced from $1.25 \%$ to $0.38 \%$. Therefore, ceramic shell with excellent properties can be prepared by adjusting the addition of mineralizer, the addition and the length of short-cut carbon fiber.
\end{abstract}

\section{Introduction}

With performance of the aerospace and industrial gas turbine blade continuing to improve, the hollow single crystal blade has been developed at home and abroad [1]. The successful preparation of the blades is based on the following characteristics: (1) Sufficient ambient temperature strength(green strength) to withstand dewaxing without failure; (2) Enough high temperature strength to withstand the static pressure of molten metal; (3) In order to improve the surface finish, the shell wall must not react chemically with the molten metal, so high chemical stability is indispensable; (4) High temperature creep resistance to ensure accuracy of shape and size of the casting; (5) Favorable permeability and thermal conductivity be aimed to improve the rate of single crystal formation.Especially[2,3,4]. Therefore, the research of the mechanical properties of ceramic shell has practical productive significance.

At present, the researches for shell of the fused corundum - silica sol binder, the domestic research scholars mainly have been adding mineralizer to the slurry to improve shell performance, Such as 811-type shell of mineralizer[5,6]. Ceramic shell be modified by P.M.Curran et al.who used $\mathrm{CaO}$ to obtain a new shell with high strength and favorable creep resistance[7]. However, the single crystal blade, with the more complex structure and the increasing size, results in that the shell is easy to be cracked due to the insufficient green strength during the process of preparation. In particular, the thickness of the sharp edge of the shell is reduced by 2 to $3 \mathrm{~mm}$ compared to the large plane, as a results, leading to the emergence of cracks and bleed-out in the directional solidification of casting[8]. Meanwhile, with the pouring temperature of the high temperature alloy continuing to increase, so non-deform ability of shell reduced that size of casting cannot be guaranteed.Lv kai et al.who added glass fiber into slurry system, the results showed that with the amount increasing of glass fiber, the bending strength of the shell increased significantly,and the high temperature gravitational distortion first increased and then decreased[9].S. Jones et al. have studied polymer modified shell and nylon fiber shell, both of which effectively improved the smoothness of the inner surface of the shell and reduced the shell thickness. The bending strength of the polymer modified shell was obviously improved than nylon fiber shell[10]. The shell was prepared by US Howmet Co.,Ltd.who used continuous carbon fiber without bleed-out and waved surface, chord width of the blade without elongation and the bow of blade without displacement[11].

According to the above investigations, the ceramic shell is studied by combining high tensile 
strength and good thermal conductivity of the carbon fiber with the modified mineralizer which be composed of kaolin and magnesia[12,13], in order to acquire ceramic shell which satisfies the process requirement of the single crystal directional solidification technology.

\section{Experimental Materials and Method}

\section{Experimental Materials and Specimen Preparation}

Corundum $\left(\alpha-\mathrm{Al}_{2} \mathrm{O}_{3}\right)$ for investment casting and the core sand mixture using alumina as refractory particle, Silica Sol as binder, and mineralizer and short-cut carbon fiber as additives.Among them, the mineralizer be composed of kaolin of $95 \%$ to $98 \%$ and magnesium of $2 \%$ to $5 \%$.short-cut carbon fiber length of $1 \sim 5 \mathrm{~mm}$, mono-filament diameter of $6 \sim 7 \mathrm{um}$.

The bending strength specimen of $70 * 30 * 6 \mathrm{~mm}$ was prepared according to the requirements, as shown in Fig.1. And the specimen was prepared for high temperature gravitational distortion of the inner diameter of $\Phi 80 * 6 \mathrm{~mm}$, as shown in Fig.2. The XQY-II intelligent sand intensity meter was used to measure the high-temperature bending strength of the shell, and the high temperature gravitational distortion was measured by the vernier caliper. And the fracture morphology was observed by scanning electron microscopy.

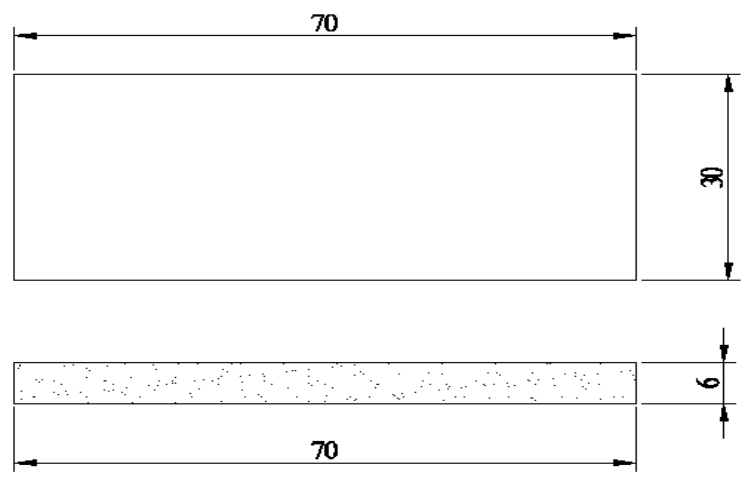

Fig. 1 Configuration of specimen used for bending strength

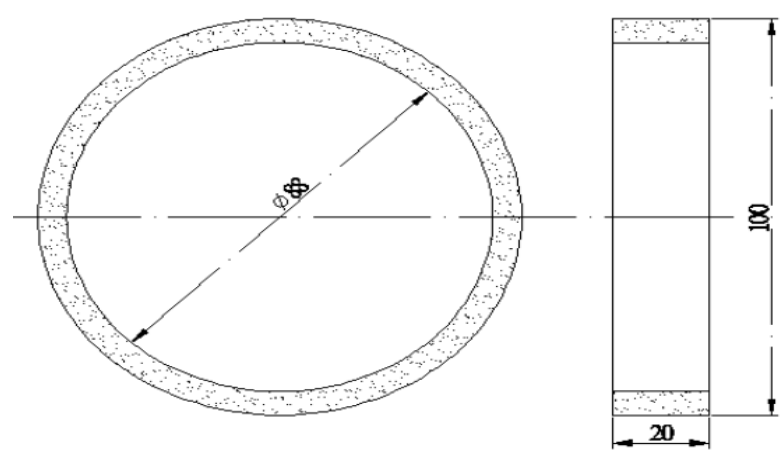

Fig.2 Configuration of specimen used for high-temperature gravitational distortion

\section{The Design of the Experimental Scheme}

In this experiment,three factors three levels orthogonal experiment was adopted[14],designing three variable factors: the length and addition of short-cut carbon fiber, and the addition of mineralizer. $\mathrm{L}_{9}\left(3^{3}\right)$ orthogonal table was designed and the level of experiments factors in Table 1.According to the analysis of the experiment results, the better level of various factors and the optimal combination was determined. On the basis analysis of range and variance to determine the impact of various factors on the test index,And mapping the trend figure of factors and index. 
Tab. 1 Level of experimental factors

\begin{tabular}{cccc}
\hline Level actor & $\begin{array}{c}\text { Length of short-cut } \\
\text { carbon fiber[mm] }\end{array}$ & $\begin{array}{c}\text { Addition of } \\
\text { mineralizer[\%] }\end{array}$ & $\begin{array}{c}\text { Addition of short-cut } \\
\text { carbon fiber [g/ml] }\end{array}$ \\
\hline 1 & A & B & C \\
2 & 1 & 2 & 0.1 \\
3 & 3 & 4 & 0.3 \\
\hline
\end{tabular}

\section{Results and Discussion}

Tab. 2 displays the orthogonal test results of the bending strength of the ceramic specimen. Analysis of experimental data can be obtained, the highest bending strength of specimen is N3 of $11.63 \mathrm{MPa}, \mathrm{N} 5$ has the lowest high temperature gravitational distortion of $0.38 \%$, compared with specimens without the mineralizer and short-cut carbon fibers (Bending strength of $7.79 \mathrm{MPa}$, high temperature gravitational distortion of $1.25 \%$ ), performances of composite modified shell has been significantly improved in these two aspects. Depending on range and variance of the orthogonal, the results showed addition of mineralizer was the greatest, addition of short-cut carbon fiber was medium, and length of short-cut carbon fiber contributed minor in the three factors affecting bending strength of specimen. The addition of short-cut carbon fiber was the greatest, length of short-cut carbon fiber was medium,and addition of mineralizer contributed minor in the three factors affecting the high temperature gravitational distortion. The optimal combination of these three factors for the bending strength and the high temperature gravitational distortion of the shell can be obtained by calculation of the orthogonal factor level, and optimal combination is $\mathrm{A} 2 \mathrm{~B} 3 \mathrm{C} 3$ and $\mathrm{A} 3 \mathrm{~B} 3 \mathrm{C} 3$, respectively.

Tab. 2 Orthogonal table of specimen performance

\begin{tabular}{cccccc}
\hline \multirow{2}{*}{$\begin{array}{c}\text { Experiment } \\
\text { al number }\end{array}$} & A & B & C & $\begin{array}{c}\text { Eending } \\
\text { strength(MPa) }\end{array}$ & $\begin{array}{c}\text { High temperature } \\
\text { gravitational } \\
\text { distortion (\%) }\end{array}$ \\
\cline { 2 - 6 } & 1 & 1 & 1 & 8.04 & 1.10 \\
N1 & 1 & 2 & 2 & 8.56 & 0.79 \\
N2 & 1 & 3 & 3 & 11.63 & 0.52 \\
N3 & 2 & 1 & 2 & 10.10 & 0.91 \\
N4 & 2 & 2 & 3 & 9.57 & 0.38 \\
N5 & 2 & 3 & 1 & 10.27 & 0.84 \\
N6 & 3 & 1 & 3 & 8.87 & 0.45 \\
N7 & 3 & 2 & 1 & 9.63 & 0.68 \\
N8 & 3 & 3 & 2 & 10.55 & 0.47 \\
N9 & & & & &
\end{tabular}

Tab. 3 statistical analysis of specimen performance

\begin{tabular}{ccccccc}
\hline \multirow{2}{*}{ Item } & \multicolumn{3}{c}{ Bending strength } & \multicolumn{3}{c}{ High temperature gravitational } \\
& $\mathrm{A}$ & $\mathrm{B}$ & $\mathrm{C}$ & $\mathrm{A}$ & $\mathrm{B}$ & $\mathrm{C}$ \\
\hline Range & 0.57 & 1.81 & 0.71 & 0.27 & 0.21 & 0.42 \\
Variance & 0.0541 & 1.9 & 0.0838 & 0.0126 & 0.0094 & 0.0302 \\
\hline
\end{tabular}

\section{Effect of Addition of Mineralizer on Bending Strength and High Temperature Gravitational} Distortion

Fig. 3 shows the effect of different addition of mineralizer on the bending strength and high 
temperature gravitational distortion. It can be found that the bending strength of shell was improved and high temperature gravitational distortion of shell reduced while the addition of mineralizer gradually increased. When the addition of mineralizer was $6 \%$, compared to shell without mineralizer, the mechanical properties of the shell were significantly improved. This is mainly because of mineralizer adding, thermal decomposition of kaolin to generate $\mathrm{Al}_{2} \mathrm{O}_{3}$ and $\mathrm{SiO}_{2}$, the former reacted with the remaining $\mathrm{SiO}_{2}$ of the coating,and the $\mathrm{Si}^{4+}$ of the latter reacted with the active components $\mathrm{Al}_{2} \mathrm{O}_{3}$ of the filler, and the reaction caused diffusion and mass transfer. Both reactions produced a secondary mullite phase of a three-dimensional network-bridged structure as the skeleton of the shell. And the formation of spinel $\left(\mathrm{MgAlO}_{4}\right)$, resulting in a large number of lattice defects, and to further promote the mullitization, so that bending strength of the shell clearly increased.Meanwhile, a small amount of glass phase were cut off from each other by plentiful secondary mullite, thus reducing high temperature gravitational distortion of the shell.

The high temperature fracture morphology of the shells with three kinds additions of mineralizer shown in Fig. 4. It can be seen that the amount of mullite phase increases with the increase of the addition of mineralizer, and the shape changes from clusters to interconnected short columns, as a result that connection strength between crystal phase was enhanced so as to improve the mechanical properties of the shell.

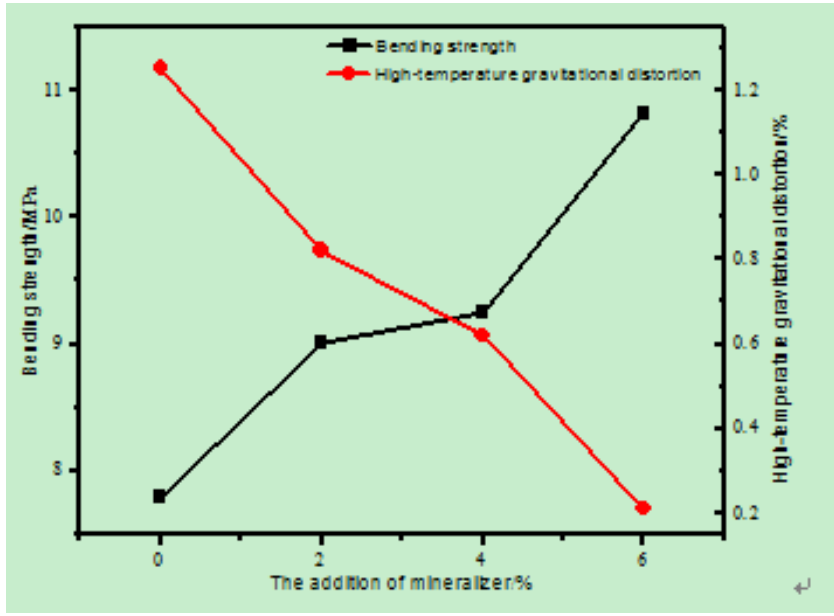

Fig .3 Effect of the addition of mineralizer on the bending strength of the shell
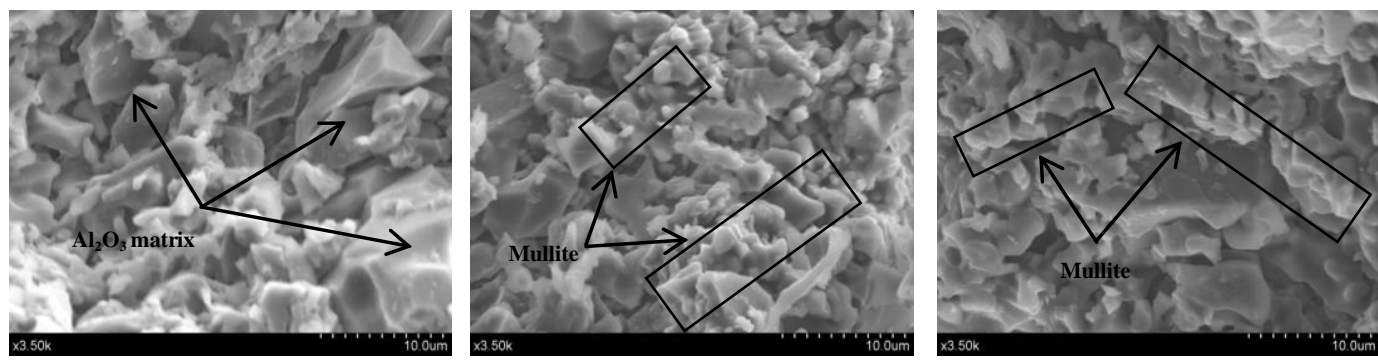

Fig.4 Fracture morphology of shell

(In a, b and c, the amount of mineralizer was $2 \%, 4 \%$ and $6 \%$, respectively)

\section{Effect of the Addition and Length of Short-Cut Carbon Fiber on Bending Strength and High Temperature Gravitational Distortion}

The addition of short-cut carbon fiber on the influence of bending strength and high temperature gravitational distortion shows in the Fig. 5. It can be found that the bending strength of the shell increased and high temperature gravitational distortion of the shell reduced while the addition of the addition of short-cut carbon fiber gradually increased. Further research showed that the toughening mechanism of the short-cut carbon fiber to the shell is fiber drawing, fiber fracture and crack turning respectively. As shown in Fig.6, the main cracks will hindered by the short-cut carbon fiber 
which distributed along the vertical direction of extension of the main cracks, and then the starting of drawing effect for carbon fiber. Due to the existence of short-cut carbon fiber, it must be to overcome the drawing work and the crack fracture work when the main crack continues to extend . Therefore, the extension of the main crack is obstructed by the short-cut carbon fibers and starts to turn in different directions, resulting in generating secondary cracks in different directions. Thus, the toughening performance of the shell can be further improved because of short-cut carbon fibers.

Fig.7 revealed the effect of the length of short-cut carbon fiber on the bending strength of the shell. As can be seen from Fig.7, the bending strength of the shell increased firstly and then decreased with the length of the short-cut carbon fiber increasing. When the length of short-cut carbon fiber (such as the length of $5 \mathrm{~mm}$ in the experiment) exceeds a certain critical value, the short-cut carbon fiber tends to bent so that be intertwined with each other and the accumulation, and resulting in the following two cases. Firstly, the short-cut carbon fiber cannot be uniformly distributed in the shell and the effective length decreased, so it cannot effectively prevent the expansion of the crack and weakens the toughness of the short-cut carbon fiber. Secondly, because the length of short-cut carbon fiber increased, so short-cut carbon fiber tend to gather together, agglomerate short-cut carbon fiber is easy to burn when the shell to be sintered, as a result micro-pores to be form in the shell shown in Fig.8. The results showed that micro-pores in the shell, on the one hand, to induce the generation of micro-cracks, and leading to reducing the high-temperature strength of shell. On the other hand, although it can be seen from Fig.8 that the high temperature gravitational distortion is continuously reduced, the decrements of high temperature gravitational distortion is remarkably slow down due to the presence of micro-pores which reduces the effective area against creep.

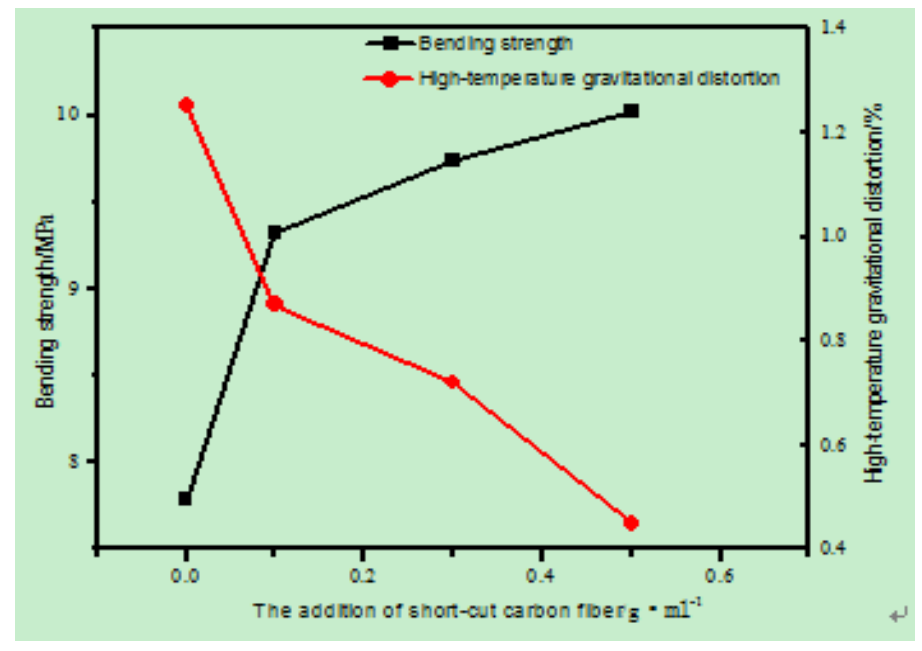

Fig. 5 Effect of the addition of short-cut carbon fiber on the bending strength

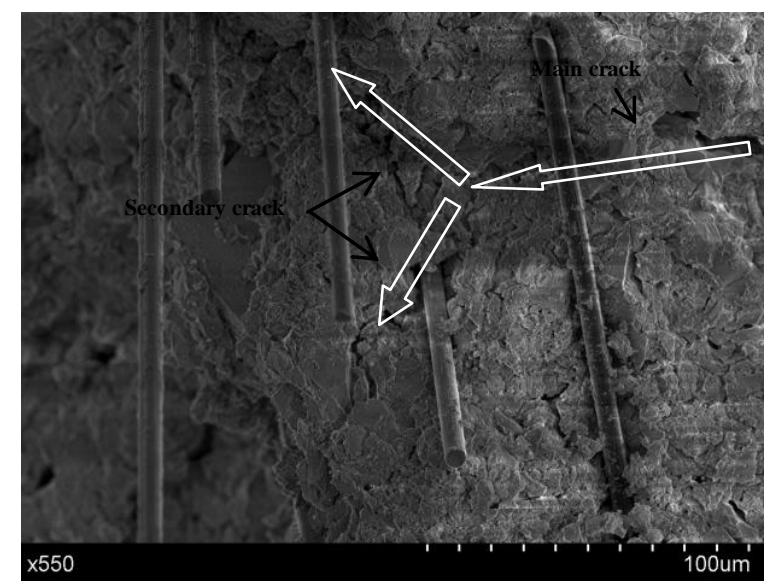

Fig. 6 Crack propagation path in composite modified shell 


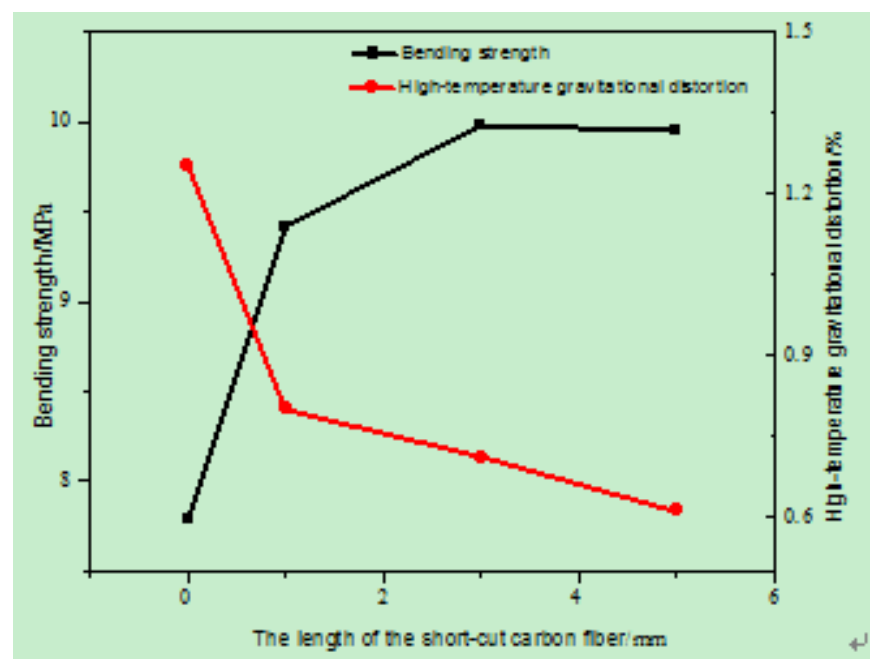

Fig.7 Effect of short-cut carbon fiber length on bending strength

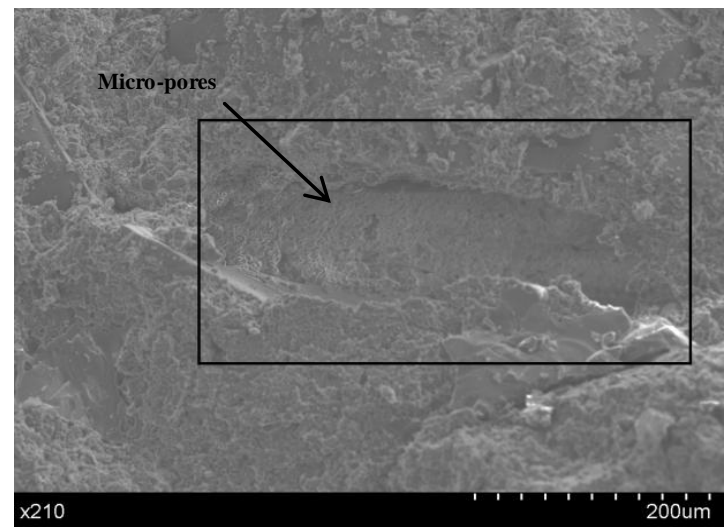

Fig.8 Micro-pore of short-cut carbon fibers after burning

\section{Summary}

1. The addition of mineralizer was the greatest, addition of short-cut carbon fiber was medium, and length of short-cut carbon fiber contributed minor in the three factors affecting bending strength of shell.

2. The addition of short-cut carbon fiber was the greatest,length of short-cut carbon fiber was medium,and addition of mineralizer contributed minor in the three factors affecting the high temperature gravitational distortion.

3. The optimum combination of mineralizer and short-cut carbon fiber to improve the bending strength and high temperature gravitational distortion of the shell is $\mathrm{A} 2 \mathrm{~B} 3 \mathrm{C} 3$ and $\mathrm{A} 3 \mathrm{~B} 3 \mathrm{C} 3$, respectively. The optimum process of composite modified shell is the addition of mineralizer of $6 \%$, the length of short-cut carbon fiber of $4 \mathrm{~mm}$ and the amount of chopped carbon fiber of $0.5 \mathrm{~g} / \mathrm{mm}$.

\section{Acknowledgements}

The author wished to thank Jiangsu Industrial Support (BE2014007-2,BE2014007-3, BE201400-4) and Jiangsu Development and Reform Commission ([2013]2027) for financial support.This work was supported by Jiangsu postgraduates innovating scientific research project (KYLX15-1061)

\section{References}

[1] Litong Zhang,Lamei Cao,Guoli Liu,Honghong Wang .Theory and Practice of Near 
Net-shape Investment Casting[M].Beijing:National Defense Industry Press, 2007, 63-115.

[2] BujuJiang,Chuanshi Li .Investment Casting[M].Beijing:China Machine Press, 2004, 2-12.

[3] Yuancai Li, Xuanpu Dong.Practical Handbook for Foundry Modeling Materials[M].Beijing: China Machine Press, 2015, 485-490.

[4] Yungfeng Chen, MooChin Wang, Minhsiung Hon. Phase transformation and growth of mullite in kaolin ceramics[J].Journal of the European Ceramic Society 2004,24,2389-2397.

[5] Jiansheng Yao, Xiaoguang Liu, DingzhongTang.et al.Effect of Proportion of Mineralizer and Concentration of Silica Sol on Properties of Ceramic Shell [J]. Rarr Metal, Materials and Enginering.2009, S2: 1222-1225.

[6] Yong Zhang.Effect of Mineralizer on Mullite Formation and Lithofacies Microstructure in Mould shell [J] .Journal Of Materials Enginering, 1997, (5): 31-33.

[7] P.M.Curran,M.H.Fassler,J.S.Perron .Calcia modified Ceramic shell mold system: CA Patent, 1080428A1[P]. 1980.

[8] Fei Wang, Fei Li,Bo He, Baode Sun, Microstructure and strength of needle coke modified ceramic casting molds[J]. Ceramics International 2014, 40: 479-486.

[9] C. Yuan, S.Jones.Advances in shell moulding for investment casting [J].Journal of Materials Processing Technology, 2003, 135: 258-265.

[10] Kai Lv Xiangdong Liu, Hao Wang,et al.Bending Strength and High temperature Self-loaded Deformation of Short Aluminum Silicate Fiher-reinforced Silicon Sol Shell[J].Journal of Materials Enginering,2015,43(07): 56-61.

[11] Xiaofu Liu, Yanchun Lou, Guiqiao Su, Bo Yu.Research Status of High Temperature Mechanical Properties of Ceramic Shell for Directional Solidification [J].2010, 30(10):0913-05.

[12] Yingchao Dong, H. Stuart,et al.Sintering and characterization of flyash-based mullite with $\mathrm{MgO}$ addition[J].Journal of the European Ceramic Society ,2011,31: 687-695.

[13] C. Yuan, S. Jones, S. Blackburn.The influence of autoclave steam on polymer and organic fibre modified ceramic shells [J].Journal of the European Ceramic Society, 2005, 25:1081-1087.

[14] Shidong Zhang, Zhongqiang Chen,JunheLiu,et al.Study on Heat Intensity of A12O3Shell Mold for Directional S o lidifica tion Casting, 2011, 60 (40): 338-340. 